\title{
A Novel Method Used to Measure the Biological Parameters via Light-Fidelity and IoT
}

\author{
Kannusamy $\mathrm{R}^{\# 1}$, Nandakumar $\mathrm{P} *^{2}$ \\ \# Head, Department of Electronics, PSG College of Arts \& Science, Coimbatore, India, \\ * Associate Professor, Department of Electronics, PSG College of Arts \& Science, Coimbatore, India, \\ ${ }^{1}$ rkpsg@hotmail.com \\ 2 nandacbe@hotmail.com
}

\begin{abstract}
Light-Fidelity (LiFi) is basically a technique to transfer data at the highest rate ever known. Using visible light for data transmission leads to many advantages and eliminates most drawbacks of transmission via electromagnetic waves. Moreover, since no interference with electromagnetic radiation occurs, visible light can be used in hospitals. Visible Light Communication (VLC) using Light Emitting Diode (LED) is a technology gives an opportunity to provide high speed and low cost wireless communication. The paper details about the measurement of bio-parameters. Mainly four kinds of measurement are done and they are ECG, Body temperature, Blood Pressure haemoglobin and $\mathrm{SpO}_{2}$ ( the amount of oxygen in the blood ) measurement. The instruments developed so far have the capability to transfer the data using wireless techniques. But here the data being analysed is transmitted via light. The most advantageous part of the instrument is that the data is transferred at the rate of light so it becomes too easy to transfer huge numbers of data within few seconds which makes it best suited for bio-medical applications. Hence the radiation effect which is one of the common problems faced by the patients is completely eradicated due to transmission using LiFi.
\end{abstract}

Keyword - LiFi, VLC, IoT, AD624AD, LED, Photodiode, Pressure sensor.

\section{INTRODUCTION}

With the exponentially increasing data demand but limited available radio spectrum, alternatives will be necessary to accommodate the needs of wire-free communication systems. This system will illustrate the problems of current wireless communication systems and alternatives to these systems, as well as motivations and possible applications for Visible Light Communications [1].

As societal dependence upon wireless systems continues to grow, wireless technology needs to expand to meet the demand. Phones, laptops, and global positioning systems are all devices that implement certain forms of wireless communication to send information to another location. However, the availability of current forms of wireless is very limited, and it is not necessarily safe to implement wireless radio, making it necessary to explore other alternatives to wireless communication to allow continued expansion upon communication systems and to ensure safe use.

In addition to the crowding of the frequency spectrum, interference is also a concern for many existing wireless systems. Any simultaneous use of a frequency band will cause interference due to the electromagnetic nature of most wireless devices, which could result in incorrect or loss of information for those users involved. A prime example of this is the use of mobile devices on planes, which directly affects safety [2].

VLC systems have more flexibility and integrity than other communication systems in many regards. Since the medium for transmission in VLC systems is visible light and not RF waves that can penetrate walls, the issue of security is inherently solved because light cannot leave the room, containing data and information in one location.

In recent years the research are going to find the alternative energy source and products. The purpose is to reduce the energy consumption, that too in lighting area. VLC uses the visible light spectrum from $380 \mathrm{~nm}$ to $780 \mathrm{~nm}$. The fast switching capability of the LED enable the data transmission [3]. VLC system based on white LEDs has emerged as an eco-friendly IT green technology using THz visible light spectrum in provision of both lighting and wireless access. Installation of new communication cables between other fixed network and LED 
lights is expensive, disruptive and time consuming process. Meanwhile, the power-line communications (PLC) can make it possible to use the power lines as the medium of communications.

The utilities of home networking over power-lines can take advantage of the existing wiring infrastructure for provision of illumination cum communication. The integrated system of VLC and PLC is a smart way of fulfilling the premise of broadband access for home networking, while providing efficient and low-cost lighting. To achieve the higher data rates (MHz), PLC channel is simulated using DMT-QAM modulation scheme [4].

VLC is a new technology that may become an alternative choice for wireless communication in the future. The technology may be used in many indoor applications. The specific presentation of VLC in this study is with the use of LEDs as the medium for transmission. Digital information will be sent through the LEDs as light pulses, and then a receiver will be able to collect the light pulses as a code and translate it to a corresponding audio data. In this study, the visible light communication system uses modulation schemes, such as on-off keying, so that the digital HIGHs and LOWs are representations of the binary code to be transmitted. Acknowledgement lights incorporated in the receiver are also utilized to make multicasting possible, and to signal that transmission is successful [5-8].

Smart phones, tablets, and the rise of the Internet of Things are driving an insatiable demand for wireless capacity. This demand requires networking and Internet infrastructures to evolve to meet the needs of current and future multimedia applications. Wireless HetNets will play an important role toward the goal of using a diverse spectrum to provide high quality-of-service, especially in indoor environments where most data are consumed. An additional tier in the wireless HetNets concept is envisioned using indoor gigabit small-cells to offer additional wireless capacity where it is needed the most. The use of light as a new mobile access medium is considered promising. In this article, we describe the general characteristics of WiFi and VLC (or $\mathrm{LiFi}$ ) and demonstrate a practical framework for both technologies to coexist. We explore the existing research activity in this area and articulate current and future research challenges based on our experience in building a proof-of-concept prototype VLC HetNet [9].

LiFi is a wireless optical networking technology that uses LEDs for data transmission. LiFi is designed to use LED light bulbs similar to those currently in use in many energy-conscious homes and offices. However, LiFi bulbs are outfitted with a chip that modulates the light imperceptibly for optical data transmission. LiFi data is transmitted by the LED bulbs and received by photoreceptors. LiFi's early developmental models were capable of 150 megabits-per-second (Mbps). Some commercial kits enabling that speed have been released. In the lab, with stronger LEDs and different technology, researchers have enabled 10 gigabits-per-second (Gbps), which is faster than 802.11ad. [10-13]

\section{A. Benefits of LiFi:}

- $\quad$ Higher speeds than Wi-Fi.

- 10000 times the frequency spectrum of radio.

- $\quad$ More secure because data cannot be intercepted without a clear line of sight.

- $\quad$ Prevents piggybacking and Eliminates neighbouring network interference.

- $\quad$ Unimpeded by radio interference.

- Does not create interference in sensitive electronics, making it better for use in environments like hospitals and aircraft.

By using LiFi in all the lights in and around a building, the technology could enable greater area of coverage than a single WiFi router. Drawbacks to the technology include the need for a clear line of sight, difficulties with mobility and the requirement that lights stay on for operation [14-16].

VLC is emerging development in latest LED technology for both illumination and for the data transmission. This has potential area in research to used in various applications. This paper presents various parameters of a human body is monitored using LiFi and the data is transmitted through IoT technique and these values are stored in Cloud. The system gives overall patient information anywhere, any time and in any device. 


\section{MATERIALS AND MethodS}

Components used are instrumentation amplifier AD624AD, high intensity LED bulbs or laser, photodiode, 8051 controller, electrodes for signal measurement, temperature sensor and pressure sensor.

This system uses VLC transmission aims to eliminate the use of RF Technology in Hospital. The information about the patients in terms of biological signals are picked up and blinked using LED's. These LED's are act as a Transmitter. All the signals are transmitted through it. These signals are received via Photo Detectors which are act as a Receiver. The received signals are analysed and stored using Internet of Things ( IoT ) in Cloud space for further use. In any emergency situation the system also gives an alert

\section{BLock Diagram For MeAsurement OF Bio-PARAMETERS}

This instrument consists of four circuits namely ECG measurement circuit, body temperature measurement circuit, pressure measurement circuit haemoglobin and $\mathrm{SPO}_{2}$ measurement circuit. All such parameters are measured one after the other with the help of selector switch. Then each signal is conditioned with the help of signal conditioner circuit and further fed to LiFi transmitter. LiFi transmitter consists of Darlington IC used for amplification and driving led for transmission of data. Fig. 1 shows the block diagram for bio parameter measurement.

Data transmission is done with the LED, here the property of Led that it has fast switching characteristics. The data is actually decoded as 1 and 0 i.e. for an LED it is either on or off. The data is transferred at the rate of light speed. Basically the rate of transmission is faster when compared to that of wireless transmission of data. And on the either side, receiver is present which is directly connected to the physicians those who want to analyse. The data is transmitted wirelessly. The photodiode senses the light rays falling onto it and fed to the stage-wise amplification circuit and then fed to the receiver pin of micro controller (8051 328) is used. Using IoT cloud of data is created and then it can be retrieved from the server whenever it is necessary. With the help of wireless transmission these data is sent to the PC and can be plotted as graph and different type of interpretation can be done.

The data "design" is transmitted with the help of LiFi. The data is first decoded to respective ASCII code and that is $68,69,83,73,71$ and 78 . Here these ASCII codes are converted to binary and also character by character. Each data is sent digitally. The data is sent in the form of square pulse. Fig. 2 explains about how the data is transmitted in this setup.

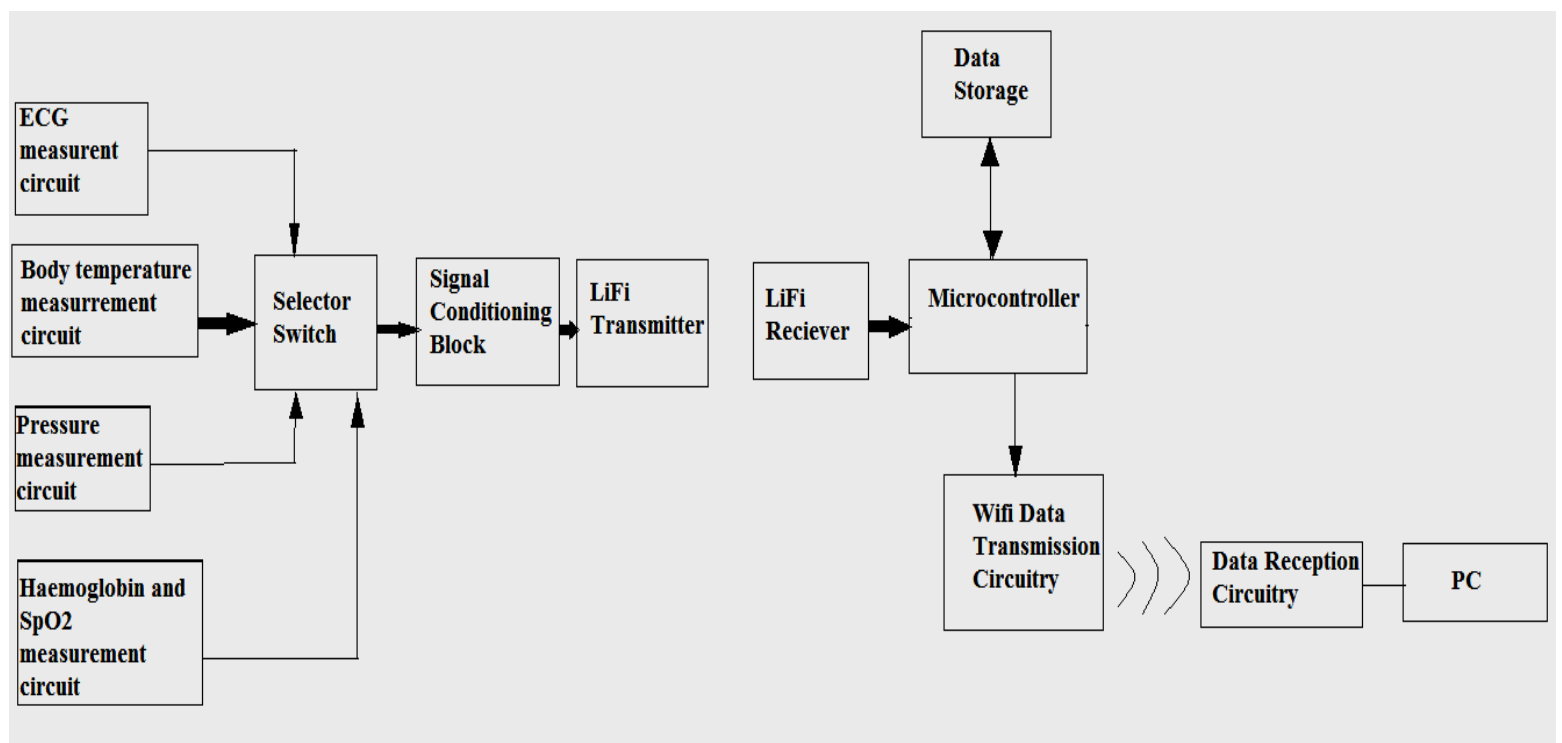

Fig. 1. Block Diagram for Bio- parameter measurement 


\section{CIRCUIT DiAgRAM EXPLANATION}

The circuit diagram consists of two parts transmitter and receiver section. Only a single parameter such as ECG measurement is shown in above Fig. 2. Likewise many such parameters like temperature, pressure and etc can be measured with the help of selector switch. Here the ECG signals is filtered and then transmitted wirelessly with LiFi technology. Fig. 3 shows the Transmission and reception circuitry

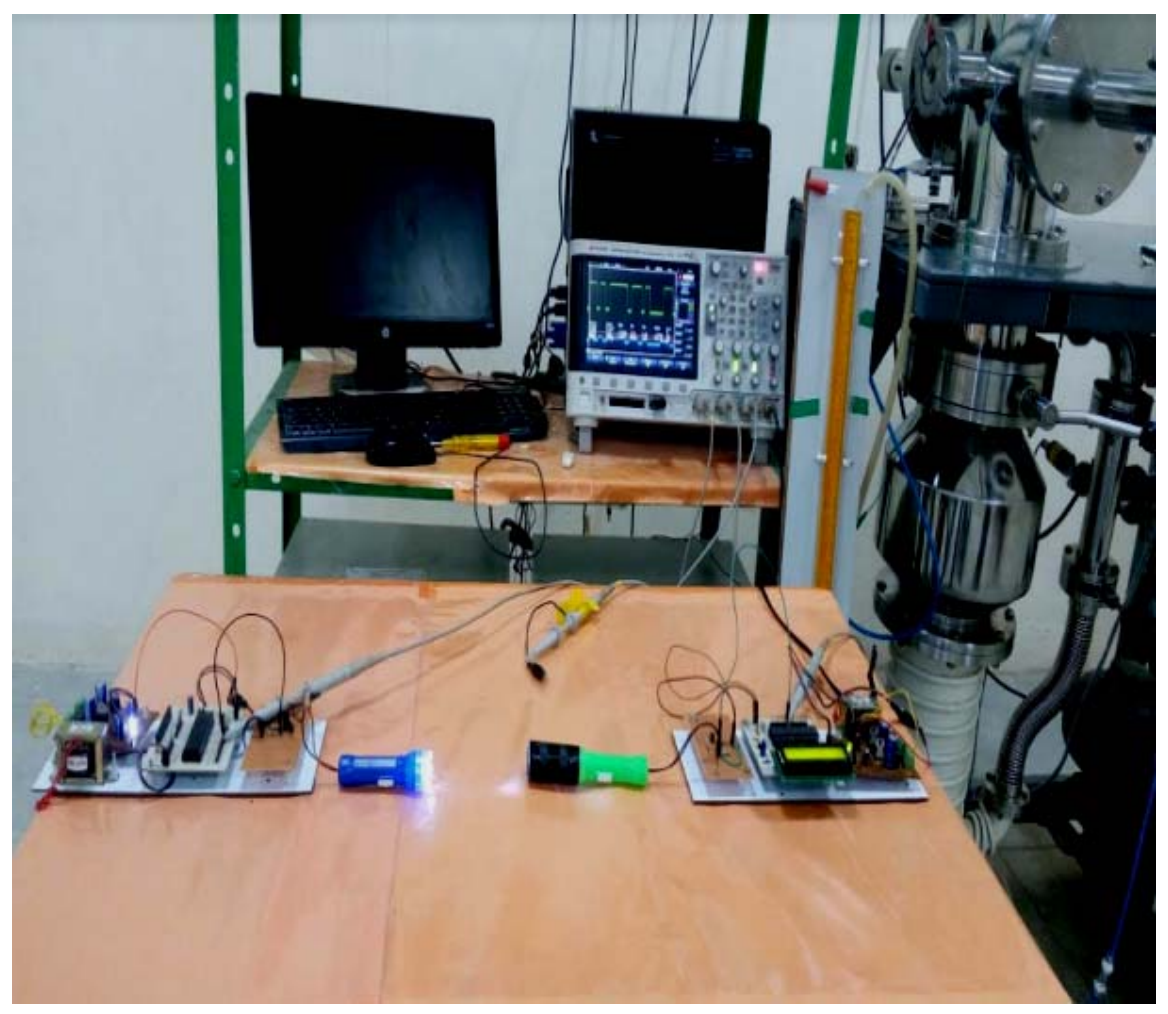

Fig. 2. Final Data Transmission Implementation in LiFi

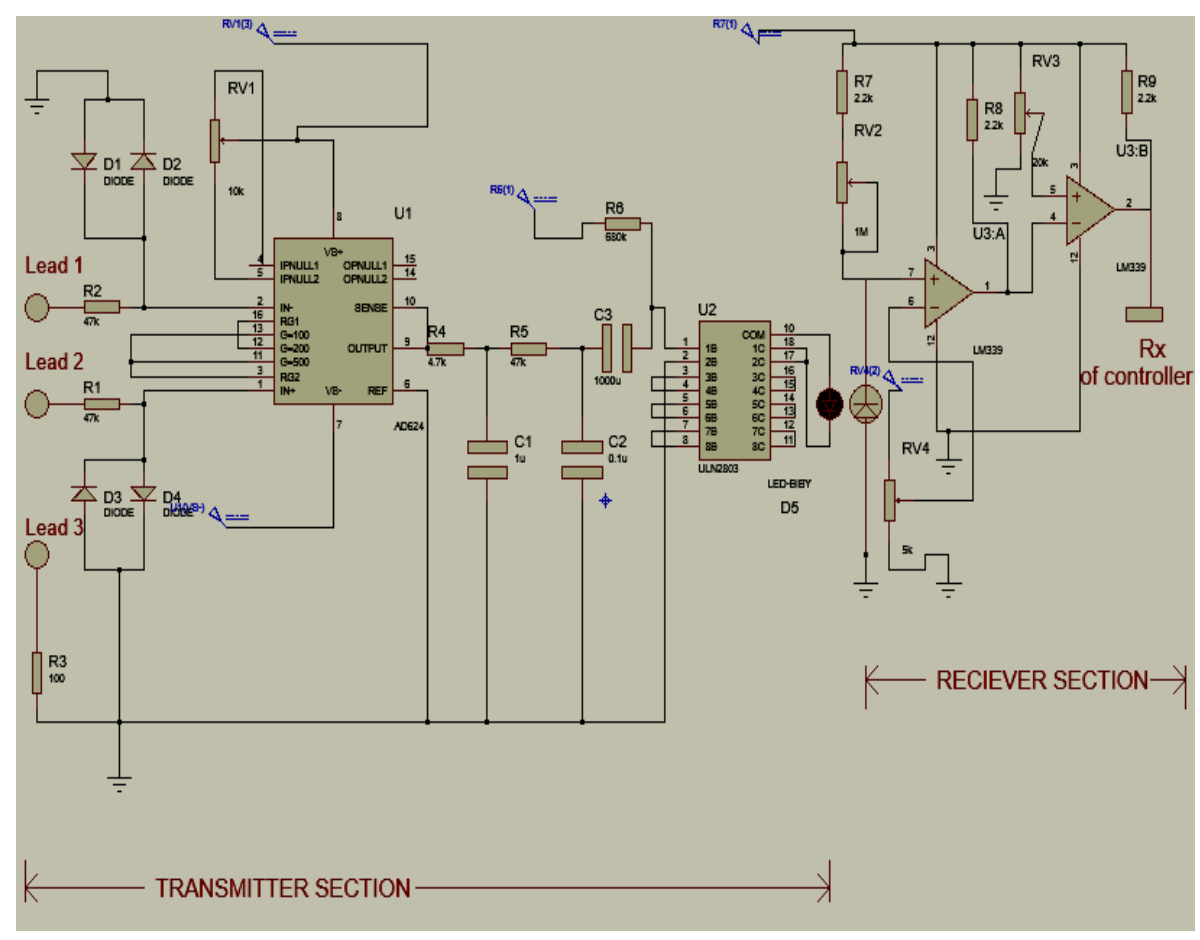

Fig. 3. Transmission and reception circuitry 


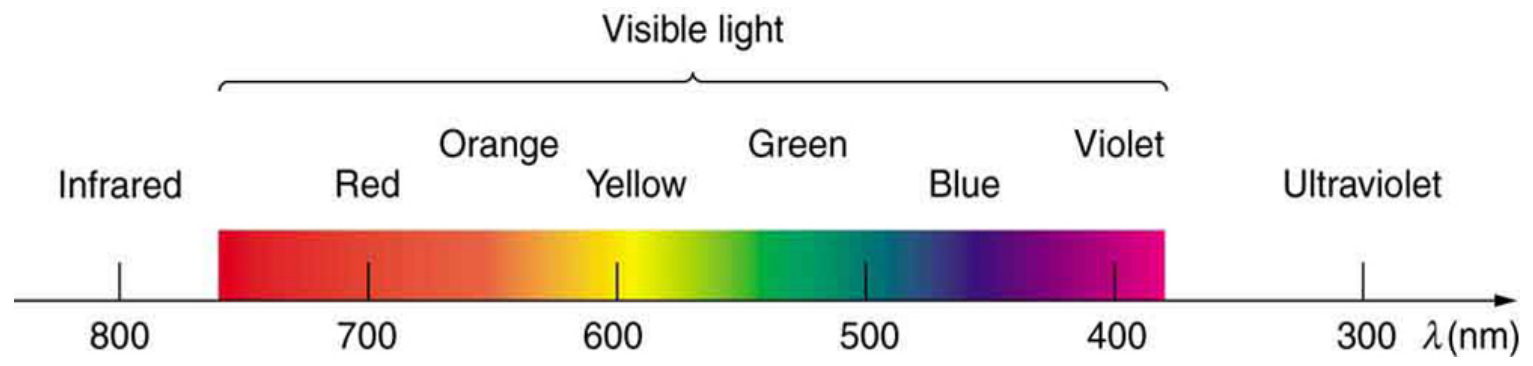

Fig. 4 Visible Light Spectrum

\section{TRANSMITTER SECTION}

This section consists of two circuitry combined and it is ECG measurement circuit and the next is LiFi transmission circuit. In this section ECG measurement circuit consists of 3 lead measurement system connected to an instrumentation amplifier AD624AD which amplifies the signal and then fed to the band pass filter. The resistance and capacitance value is chosen such a way that it acts as band pass filter. It is used in variety of application. The signal of required frequency is given to the Darlington IC for transmission of data with the help of LED. This IC decodes the data to binary for transmission. Here on state of led is represented as 1 and off state of LED is represented as 0 . So while transmission, the data is sent as 0 or 1.

\section{RECEIVER SECTION}

This section consists of LiFi receiver. In this circuit it consists of mainly amplifiers and photodiode. Photodiode senses the light falling onto it and produces the voltage proportional to it. This voltage is sensed by two stage amplifier and then it finally reaches the receiver pin of the micro-controller. Microcontroller accesses these data and then converts signals to values and then stored in cloud. Either by using MATLAB or LABVIEW each data is interpreted as graph and stored in PC. Whenever this data is needed, it can be accessed from the cloud with the help of Wi-Fi technology.

\section{IMPLEMENTATION OF LIFI TECHNOLOGY}

Visible light should be considered as the medium for wireless transmission because it has a few advantages over other standard wireless transmissions. The first reason to consider is visible light's frequency spectrum bandwidth as shown in Fig.4. which ranges from $430 \mathrm{THz}$ to $750 \mathrm{THz}$. The bandwidth is much larger than the radio frequency bandwidth, which ranges from $3 \mathrm{kHz}$ to $300 \mathrm{GHz}$. With a larger bandwidth it is possible to accommodate more users and potentially achieve higher transfer rates because each user can be given a larger portion of the bandwidth to transfer information.

If the communication system will be used in hospitals, the transmissions will not occur in the Industrial, Scientific, and Medical (ISM) band, therefore not interfering with medical devices. On top of having a higher bandwidth, the frequency spectrum has less regulation than the radio spectrum. With little regulation, the user will be able to choose any frequency to transfer information. If visible light communication systems become more popular, regulations could be placed on these forms of data transmission for the same reasons that they were placed for the radio spectrum.

The proposed system is consist of the following modules i) sensor module ii) data reading module iii) data convention module iv ) Transmitter module v) Receiver module vi ) Microcontroller module vii ) storage viii ) Data interpretation module ix ) IoT module $\mathrm{x}$ ) PC 


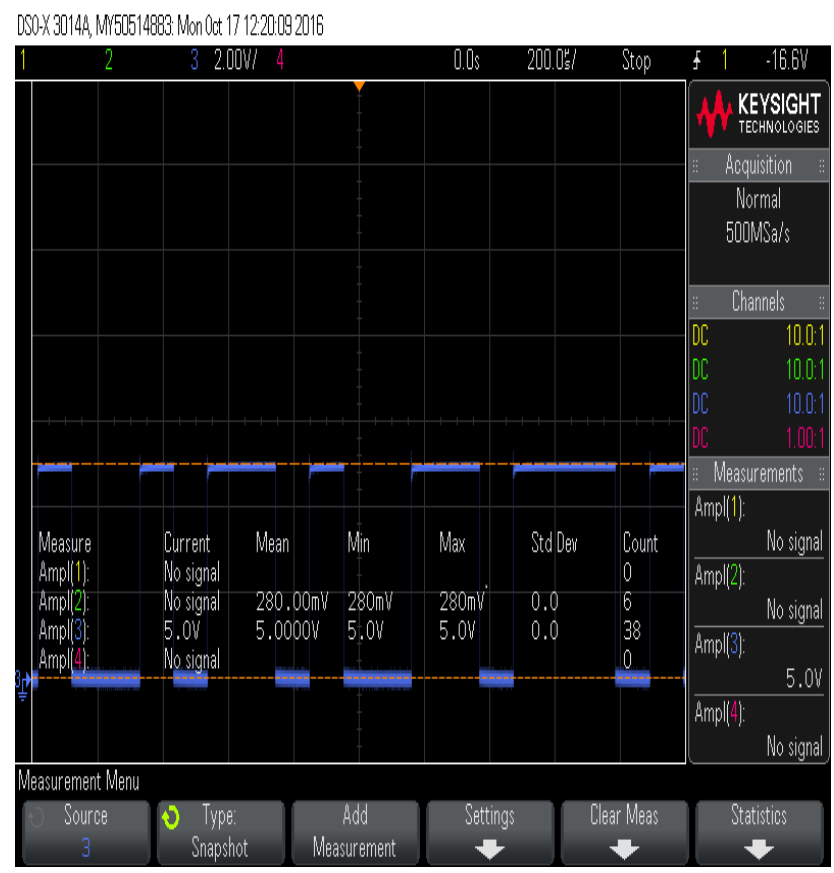

Fig. 5. Data in Square wave transmission

The reliability of the proposed system are tested by carried number of experiments. This VLC system is configured for unidirectional data transmission of bio medical signals. Basically the research consists two major sections. Firstly the data transmissions as well as reception without loss of signal strength using LiFi technology. Then the basic bio-parameter measurement had done with the basic circuitry involved.

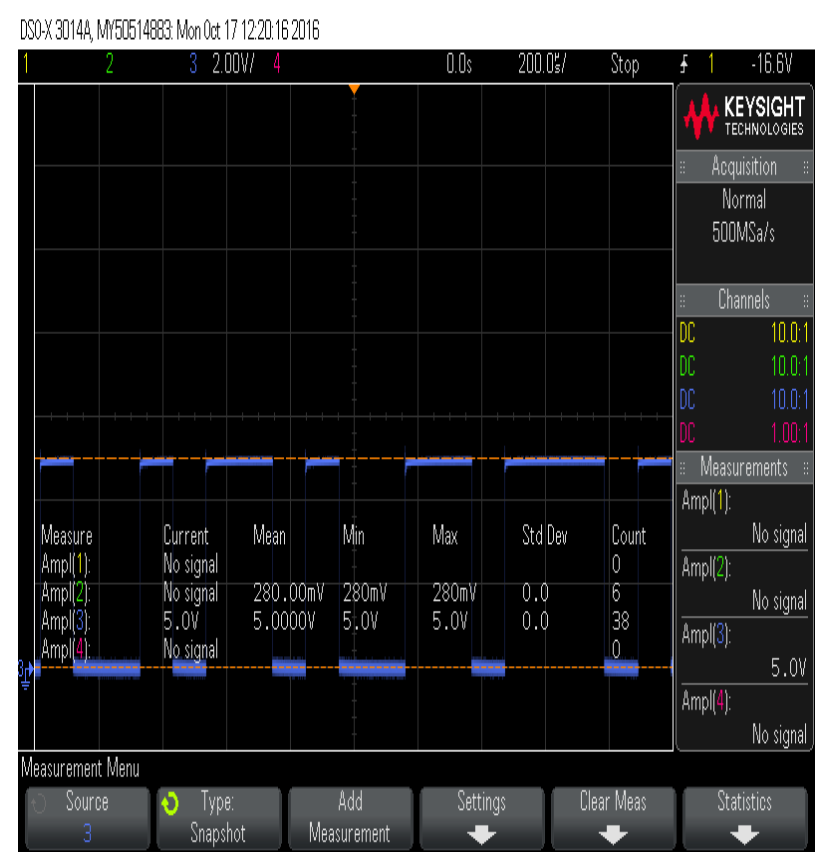

Fig. 6. With different voltage level

These two sections are combined together to yield the particular result. This section explains how different data is transmitted and how it is received and how the square waveform changes with data change. Moreover even it can be observed that the signal sent to the transmitter is exactly retrieved from the receiver. 
Fig. 5 and Fig. 6 shows the data being transmitted and the image being taken from the oscilloscope. The voltage measured is also informed in the graph. It is basically a square wave with minimum voltage as $280 \mathrm{mV}$ and maximum limit as $5 \mathrm{~V}$. The data input is represented here.

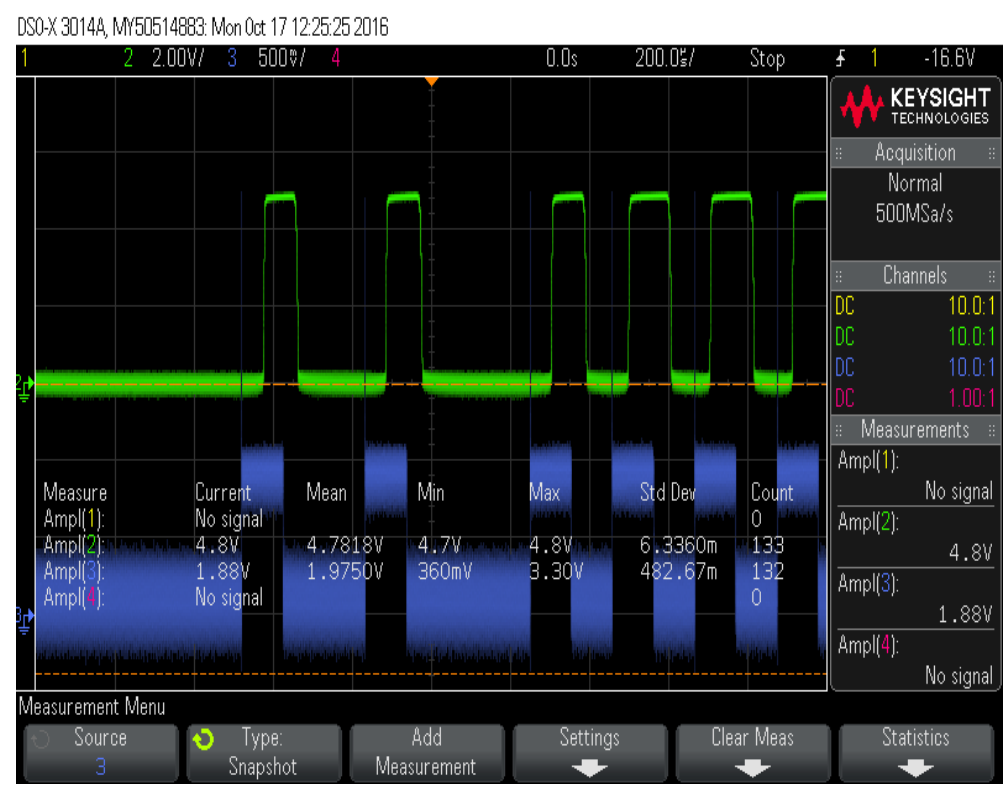

Fig. 7. Two wave combination different and transmission

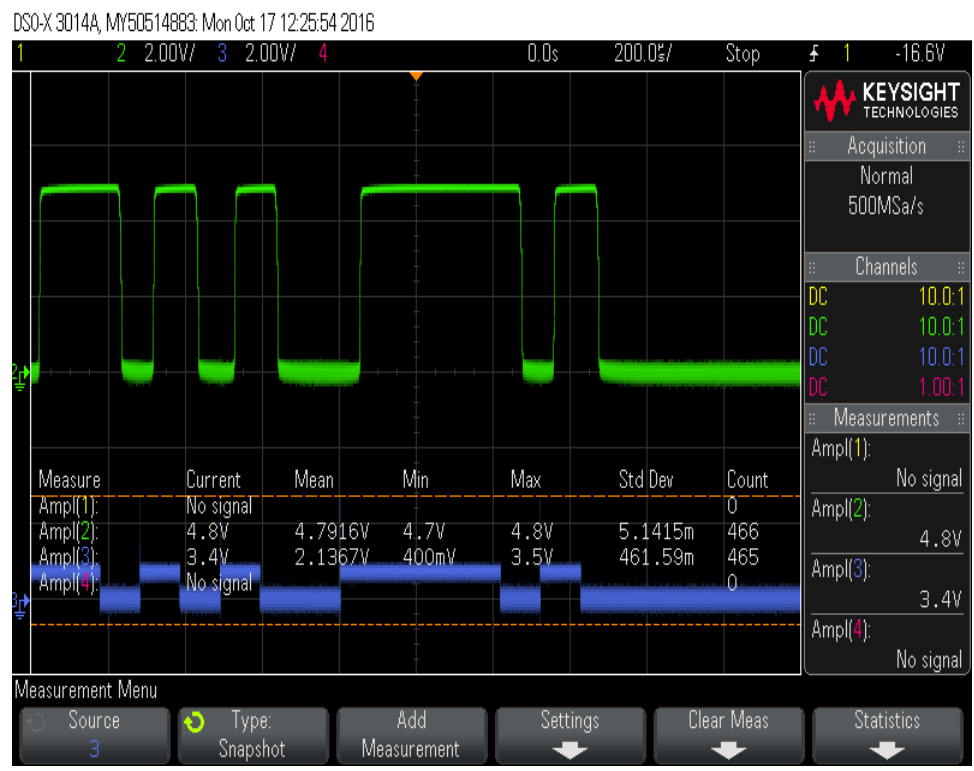

Fig. 8. Two different square wave at voltages

Fig. 7, Fig. 8 and Fig. 9 shows different level of signal which is represented in blue and green. The level of two signals is completely different. They are different in amplitude and moreover the maximum and minimum limit also differs. The amplitude of the blue signal is decreased from voltage to millivolts.

This is compared with the reference signal that is the input signal. The image is captured from both the input as well as output that are transmitter as well as receiver. The data transmitted is represented as blue and the data received is represented as green. It is analysed that the sent to the receiver is much like the data transmitted. 
Fig. 10 and Fig 11 clearly depicts the data being transmitted and the data being received in LCD display which is also interfaced with the transmitter. Firstly the data is converted to ASCII values and then decoded to binary which is actually on and off of an LED which is sensed by the photodiode and then the data is again converted to the hex code and sensed by the microcontroller. Controller helps in displaying the output.

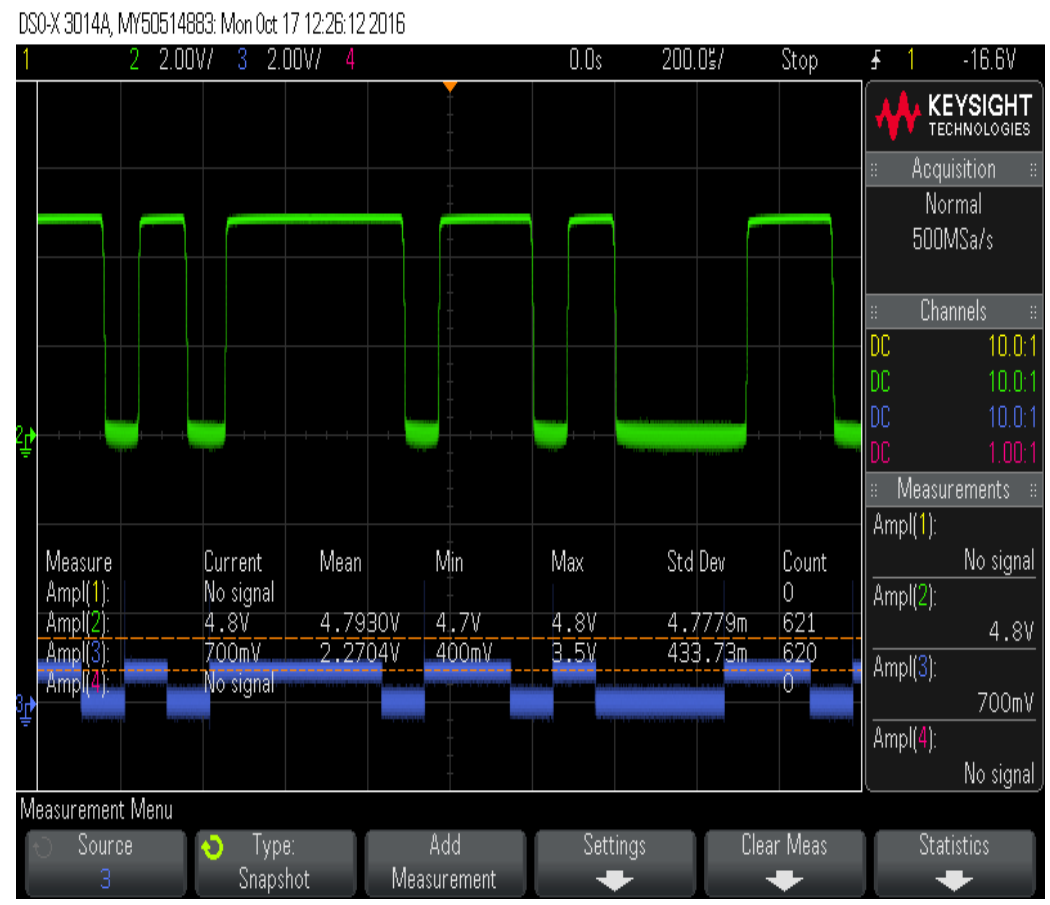

Fig. 9. Two different level of signal with different mean

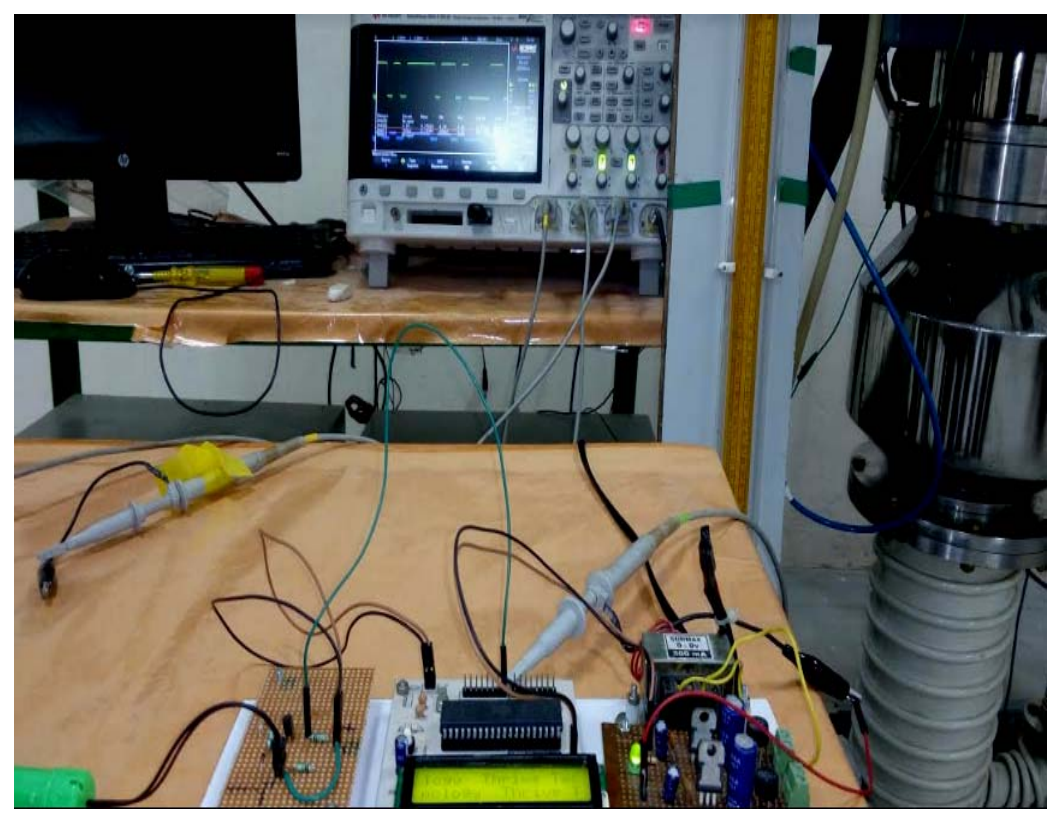

Fig. 10. Receiver and CRO image 


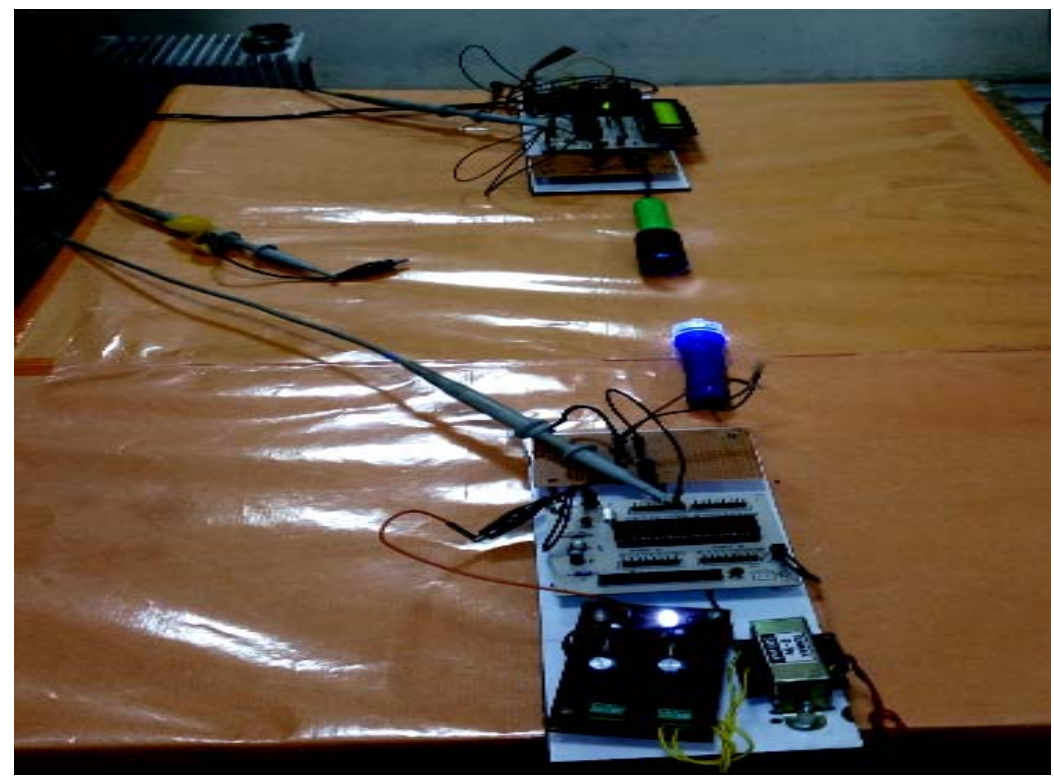

Fig. 11. Transmitter and Receiver Interface

\section{CONCLUSION}

The system proposed in this paper is capable of transmitting data between two devices at the speed of a few kbps. The main requirement is line of sight between the transmitter and receiver. Hence this system can be used in closed area or room. LiFi technology is still in the starting phase. Indoor networking and location based products are slowly available in the market. LiFi is expected to do more in IoT, Smart Cities, Power over Ethernet (PoE), Wireless Sensor Networks (WSN) etc. LiFi is depends strongly on special LEDs and Photo Diodes or Image sensors.

This system exhibits the biomedical signals of patient information are transmitted via visible light. With the help of latest internet speed and devices the proposed system enables online monitoring of the patient's condition. And the data is transmitted to anywhere in the world for study, analysis or to report. And also short messages can also send to notify persons in case of abnormal conditions. There are so many bio-medical instruments which operate at different specifications. But the most important of all is that nowhere the instrument communicates with light. Here, this instrument has the capability to communicate with the speed of light. The important aspect is that no radio wave interference is caused. Radio waves can too affect the patients. The wiring around them too causes the discomfort. All these issues are sorted out and the communication is made better. The analysis rate is also improved. More number of patients are analysed within less span of time. Technicians feel more comfortable to handle such kind of instrument.

\section{FUTURE WORK}

In future one can use microLED's for data transmission which flickers at a higher data rate than normal LED's. A cluster of LEDs can be used for parallel data transmission. LED's of different colours can be used to enhance the data transmission rate. LiFi technology can perform a critical role in providing superfast home broadband internet that will lead to higher bandwidth data communication on different platforms at the same time. LiFi takes VLC further by using LEDs to realise fully networked wireless systems. Synergies are harnessed as lights become LiFi attocells resulting in enhanced wireless capacity for the IoT, 5G and beyond. 


\section{REFERENCES}

[1] Harald Haas, "What is LiFi?” European Conference on Optical Communication pp: 1 - 3, 2015.

[2] Moussa Ayyash; Hany Elgala; Abdallah Khreishah; Volker Jungnickel; Thomas Little; Sihua Shao; Michael Rahaim; Dominic Schulz; Jonas Hilt; Ronald Freund "Coexistence of WiFi and LiFi toward 5G: concepts, opportunities, and challenges" IEEE Communications Magazine, pp: 64-71, 2016.

[3] U. S Department of Energy, Part I : Review of Life-Cycle Energy Consumption of Incandescent, Compact Fluorescent, and LED Lamps, 2013.

[4] Krishna Prasad Pujapanda "LiFi Integrated to Power-lines for Smart Illumination Cum Communication" International Conference on Communication Systems and Network Technologies, pp: $875-878,2013$.

[5] Adoptante E. B; K. D. Cadag; V. R. Lualhati; Mark Lorenze D. R. Torregoza;Alexander C. Abad "Audio multicast by Visible Light Communication for location information for the visually impaired" International Conference on Humanoid, Nanotechnology, Information Technology,Communication and Control, Environment and Management, pp: 1 - 6, 2015.

[6] Chung-Yi Li; Hai-Han Lu; Ting-Chieh Lu; Wen-Shing Tsai; Bo-Rui Chen; Chien-An Chu; Chang-Jen Wu; Chen-Hong Liao "A 100m/40Gbps 680-nm VCSEL-based LiFi transmission system" Conference on Lasers and Electro-Optics pp: 1 - 2, 2016.

[7] Wen-Shing Tsai; Hai-Han Lu; Chung-Yi Li; Ting-Chieh Lu; Chen-Hong Liao;Chien-An Chu; Peng-Chun Peng."A 50-m/40 Gb/s 680-nm VCSEL-Based FSO Communication" IEEE Photonics Journal , 2016.

[8] Kun Chen Hu; Ana García Armada; Matilde Sánchez-Fernández; Antonio Royo "Prototyping and measurements for a LiFi system" IEEE Sensor Array and Multichannel Signal Processing Workshop, pp : 1-5, 2016.

[9] Hai-Han Lu; Chung-Yi Li; Hwan-Wen Chen; Chun-Ming Ho; Ming-Te Cheng; Zih-Yi Yang; Chang-Kai Lu "A 56 Gb/s PAM4 VCSEL-based LiFi transmission with two-stage injection-locked technique" IEEE Photonics Journal pp: 1-1, 2016.

[10] Sihua Shao; Abdallah Khreishah; Michael B. Rahaim; Hany Elgala; Moussa Ayyash; Thomas D. C. Little; Jie Wu "An Indoor Hybrid WiFi-VLC Internet Access System" IEEE 11th International Conference on Mobile Ad Hoc and Sensor Systems, pp: 569 $574,2014$.

[11] Eyal Ronen; Adi Shamir “Extended Functionality Attacks on IoT Devices: The Case of Smart Lights" IEEE European Symposium on Security and Privacy pp: $3-12,2016$.

[12] Chyi-Da Yang; Po-Hsun Lei; Der-Jin Pong; Ming-Yuan Wu; C. -L. Ho; Wen-Jeng Ho; Meng-Chyi Wu; Keh-Yung Cheng "Edgecoupled InGaAs PIN photodiode with a light funnel waveguide" IEEE Journal of Quantum Electronics Year, pp 1607 - 1613, 2004.

[13] Víctor Monzón Baeza; Matilde Sánchez-Fernández; Ana García Armada; Antonio Royo "Testbed for a LiFi system integrated in streetlights" European Conference on Networks and Communications, pp:517-521, 2015.

[14] Richard M. Dorward; Paul A. Callan "Economic considerations for the electronic control of next generation multi-channel optical transceivers for the access network" , 14th International Conference on Transparent Optical Networks, pp :1 - 5, 2012.

[15] Hany Elgala; Thomas D. C. Little "Polar-based OFDM and SC-FDE links toward energy-efficient Gbps transmission under IMDD optical system constraints" IEEE/OSA Journal of Optical Communications and Networking, pp :A277 - A284, 2015.

[16] Zimu Zhou; Zheng Yang; Chenshu Wu; Wei Sun; Yunhao Liu "LiFi: Line-Of-Sight identification with WiFi” IEEE INFOCOM IEEE Conference on Computer Communications, pp: 2688 - 2696, 2014.

\section{AUTHOR PROFILE}

Dr. R. Kannusamy obtained his B.Sc., Physics from Kandasamy Kandars College, Velur in 1982 and M.Sc., Physics from NGM College, Pollachi in 1984 and also M.Phil., in Physics from Bharathiar University, Coimbatore in 1986. He completed Ph. D., in the year 2009. He has been working in the teaching field for about more than 25 years. He has published many articles in the reputed national and international journals. He has guided many students at PhD level.

P. Nandakumar obtained his B.Sc., Electronics and M.Sc., Applied Electronics from PSG College of Arts and Science, Coimbatore in 1996 and 1998. First Rank holder in M.Sc. and also M.Phil., in Electronics from Bharathiar University. He has been working in the teaching field for about 19 years. His area of interest is Embedded System. 